Original article

\title{
Measuring costs of community mental health care in Italy: A prevalence-based study
}

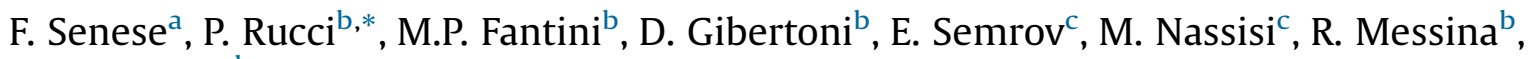 \\ C. Travaglini ${ }^{\mathrm{d}}$

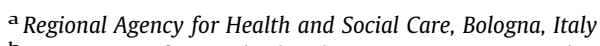 \\ ${ }^{\mathrm{b}}$ Department of Biomedical and Neuromotor Sciences, Alma Mater Studiorum University, Bologna, Italy \\ ${ }^{\mathrm{c}}$ Department of Mental Health and Pathological Dependences, Reggio Emilia, Italy \\ d Department of Management, Alma Mater Studiorum University, Bologna, Italy
}

\section{A R T I C L E I N F O}

\section{Article history:}

Received 5 October 2017

Received in revised form 13 January 2018

Accepted 9 February 2018

Available online 4 March 2018

\section{Keywords:}

Mental health costs

Direct costs

Psychosis

Community mental health services

\begin{abstract}
A B S T R A C T
Background: Information on individual mental healthcare costs and utilization patterns in Italy is scant. We analysed the use and the annual costs of community mental health services (MHS) in an Italian local health authority (LHA). Our aims are to compare the characteristics of patients in the top decile of costs with those of the remaining $90 \%$, and to investigate the demographic and clinical determinants of costs. Methods: This retrospective study is based on administrative data of adult patients with at least one contact with MHS in 2013. Costs of services were estimated using a microcosting method. We defined as high cost (HC) those patients whose community mental health services costs place them in the top decile of the cost distribution. The predictors of costs were investigated using multiple linear regression. Results: The overall costs borne for 7601 patients were 17 million $€$, with HC accounting for $87 \%$ of costs and $73 \%$ of services. Compared with the rest of the patients, HC were younger, more likely to be male, to have a diagnosis of psychosis, and longer and more intensive MHS utilization. In multiple linear regression, younger age, longer duration of contact with MHS, psychosis, bipolar disorder, personality disorder, depression, dementia and Italian citizenship accounted for $20.7 \%$ of cost variance.

Conclusion: Direct mental health costs are concentrated among a small fraction of patients who receive intensive socio-rehabilitation in community services. One limitation includes the unavailability of hospital costs. Our methodology is replicable and useful for national and cross-national benchmarking.
\end{abstract}

(C) 2018 Elsevier Masson SAS. All rights reserved.

\section{Introduction}

The recent Mental Health Action Plan 2013-2020, promoted by the 66th World Health Assembly [1], included among its main objectives the provision of comprehensive, integrated mental health and social care services in community-based settings, the implementation of strategies for promotion and prevention; and strengthened information systems, evidence and research. Mental health care comprises the main care domains provided to citizens with mental illness and/or substance use disorders (ambulatory, home, residential and semi-residential care) and accounts for 5\% of total health care expenditure in Italy, remaining a neglected area of health policy compared to other countries [2]. The recognition of

\footnotetext{
* Corresponding author at: Department of Biomedical and Neuromotor Sciences, Unit of Hygiene, Public Health and Biostatistics, Alma Mater Studiorum University of Bologna, Via San Giacomo 12, 40126 Bologna, Italy.

E-mail address: paola.rucci2@unibo.it (P. Rucci).
}

the complexity, multifactorial nature of mental illness has led to redefining the essential levels of care [3] for mental health and pathological substance dependence in Italy, with a focus on the assessment of health and social care needs, accessibility, continuity of care and personalized care pathways. To address this new challenge, in the last few years the 'health budget' has been introduced in some Italian regions as a new tool to manage the complex problems of patients with mental illness, in which the local and health care institutions, together with other resources (voluntary associations, cooperation, family) and the person himself, share pathways built on subject's needs. For each patient a personalized health budget is defined that brings together the economic, social, personal and context resources [4,5]. Still, the implementation of personalized care pathways tailored to patients' needs would require a careful assessment of feasibility and sustainability of interventions over time. Moreover, no finegrained data on activity-based budgeting of mental health services are available to support decision-making because estimates of mental health care costs are based on average costs obtained as the 
ratio between the overall costs of hospital and community care and the resident population [6].

Amaddeo et al. [7] reviewed the few existing studies conducted on the analysis of the costs of the Italian mental health provision of care and described the current financing system for mental health care in Italy.

These costs are largely borne by the national health system through the Local Health Authorities (LHAs), by patients' families and by a part of the municipality budget devoted to social care. LHA costs can broadly be divided into: costs sustained within the accounting year by the Mental Health and Pathological Dependence Department (MH-PDD) as part of the per-capita quota allocated to the Department and those generated by patients' admissions to other departments and services (ambulatory consultations, drugs, emergency, hospitalization, day-hospital and long-term rehabilitation among public and private facilities, homecare).

The MH-PDD administers approximately $5 \%$ of the $1.940,61 €$ percapita average regional expenditure in 2011-2012 [8] and its financing is granted to each LHA according to historical expenditure as a quota of the per-capita transfer made by the Region to the LHAs.

The current accounting schemes leave little room for measurement and provides no useful insight on individual care needs. $\mathrm{MH}-$ PDD costs are merely benchmarked yearly as the proportion of MH-PDD costs on the overall per-capita quota: an average $€ 82.4$ for $\mathrm{MH}$ and an average of $€ 16.68$ for PDD per-capita in 2011 . Moreover, $\mathrm{MH}$ information systems and the LHA accounting systems are not yet integrated, hindering research on individual and organizational sources of variability [9-11].

In the attempt to fill this knowledge gap, and given the lack of information on individual costs of mental illness, we set up a costing methodology to estimate the costs of 45 types of services provided by the MH-PDD. A simple bottom-up procedure was used, based on the principle of full absorption costs, that includes fixed and variable costs. The cost items included in the procedure were the workforce costs, the indirect costs associated with clinical activity and the general administration overheads allocated to $\mathrm{MH}$ PDD at 2013 prices. After linking the mental health information system and the current accounting system of a Local Health Authority in Italy, we applied this methodology to estimate the overall annual $\mathrm{MH}$ costs borne by the LHA.

Converging evidence indicates that a small proportion of mental health care users account for a large share of health care costs [12-14].

Our aims are therefore to compare the characteristics of patients in the top decile of costs with those of the remaining $90 \%$, and to analyse the demographic and clinical determinants of costs.

\section{Methods}

\subsection{Overview and data source}

The study population consists of adult patients who had at least one contact with the mental health services of the Local Health Authority of Reggio Emilia (Italy, 535869 inhabitants at 01.01.2013) in 2013.

Data on patient characteristics and on the MH-PDD services they received in 2013 were extracted from mental health information system database (SISM) database. SISM includes information on socio-demographic characteristic, on ICD-9 CM diagnoses and on the number and type of services delivered. ICD-9 $\mathrm{CM}$ diagnoses are recorded at the first/second visit by the psychiatrists/psychologists when a specific treatment plan is defined, targeted to the patient's needs and are updated over time if they change or when additional symptoms emerge. The algorithm for the classification into diagnostic groups is provided as Supplementary material.
Data are entered in the SISM on a regular basis by the mental health services operators and delivered to the Regional Health Authority for quality check twice a year. Inconsistencies are resolved and the Region, in turn, sends the data to the Ministry of Health for reporting. The SISM glossary (2013) defines 45 types of community services (Table 1 ), from the fulfillment of legal and organizational requirements (e.g. writing and exposing a report for the justice), not involving any contact with the patient, to group activities. The 45 services were classified into 7 clinicaltherapeutic pathways that included initial (re)assessment, psychotherapy, psychiatric-clinical encounter, vocational training, socio-rehabilitation, day center (DC) and community dayhospital (CDH).

Specifically, socio-rehabilitation consists of individual or group daytime activities, tailored to the needs of care or rehabilitation of patients with a high degree of disability/chronicity. Sociorehabilitation activities are aimed at providing patients social and/or economic support, or helping them (re) acquire daily activity skills and interpersonal functioning. They are carried out outside day centers or day hospital.

Day center activities are daytime group rehabilitative therapeutic activities for patients with severe mental illness.

Community day hospital interventions include drip, and parenteral or oral drug administration to patients in an acute illness phase, as an alternative to hospitalization. They are carried out in non-hospital facilities.

\subsection{Cost calculation}

The costing methodology used in the present study was based on a microcosting (bottom-up) approach to establish the costs of services at individual level.

The LHA accounting system was used to determine costs that are directly driven by service user care (clinical care staff time and the administered drugs) and those that are more loosely tied to service user activity (indirect costs and overheads).

Cost of labor estimates for each activity provided were possible because the MH-PDD database reports reliable information on the number and qualification of health professionals involved in each service and, to some extent, information regarding the duration and the location of the activity. The beginning and the end of each activity was estimated in a sub-sample of 19,634 records obtained by excluding services with zero or over $3 \mathrm{~h}$ duration. The average length in minutes for each MH-PDD activity was calculated and then validated by two experienced senior psychiatrists and a 'displacement' multiplication factor was assigned to services provided outside the typical setting (e.g. prison, patient home and acute service consultations by MH-PDD staff).

Gross hourly costs were attached to each service provided by the MH-PDD in 2013 up to a maximum of 4 health professionals involved in each activity, then multiplied by the duration of the service. The duration in minutes was set to the validated standard time (Table 1) when provided in the main location, otherwise a weight varying between $1.25-1.50$ was applied if provided in other locations requiring personnel displacement. Group activities were assigned a 0.36 weight, assuming that on average 2.8 users took part in each group activity.

Because services are mainly relational and labor-intensive, only staff costs were considered as direct costs and were derived from actual labor costs in the LHA (Table 2).

Indirect costs and overheads were taken from the LHA accounting system by selecting only those items referring to support clinical activities and MH-PDD general management. Among direct health care goods, pharmaceuticals and charges paid for buying health and rehabilitation services outside the MH-PDD 
Table 1

Mental health unit services: skill-mix, venue and average length of service weights.

\begin{tabular}{|c|c|c|c|c|c|c|}
\hline Service & $\begin{array}{l}\text { Average } \\
\text { number } \\
\text { of staff }\end{array}$ & $\begin{array}{l}\text { Team- } \\
\text { mix }\end{array}$ & $\begin{array}{l}\text { Av. } \\
\text { length }\end{array}$ & Main venue & Secondary venues & $\begin{array}{l}\text { Other venue - group } \\
\text { activity weight }\end{array}$ \\
\hline Medical assessment & 1.2 & MD & $40^{\prime}$ & $\mathrm{MHC}$ & $\begin{array}{l}\text { Comm-LHT. Prison. } \\
\text { HOME }\end{array}$ & 1.5 \\
\hline Compulsory health assessment & 1.5 & MD-N & $40^{\prime}$ & $\mathrm{MHC}$ & HOME & 1.5 \\
\hline Informal care $<4 \mathrm{~h}$ & 2.2 & N-RT & $150^{\prime}$ & $\mathrm{DC}$ & - & 0.36 \\
\hline Informal care $>4 \mathrm{~h}$ & 2.4 & $\mathrm{~N}$ & $330^{\prime}$ & DC & - & 0.36 \\
\hline Internistic assessment & 2.4 & $\mathrm{~N}$ & $30^{\prime}$ & DC & MHC & 1 \\
\hline Group discussion & 2.2 & $\begin{array}{l}\text { N-OSS- } \\
\text { OTA }\end{array}$ & $55^{\prime}$ & DC & - & 0.36 \\
\hline Family interview & 1.2 & MD-N & $50^{\prime}$ & $\mathrm{MHC}$ & HOME, Prison & 1.5 \\
\hline Phone call $>15 \mathrm{~min}$ & 3.8 & $\mathrm{~N}-\mathrm{Ot}$ & $30^{\prime}$ & MHC & - & \\
\hline Individual interview/consultation & 1.3 & MD-N & $60^{\prime}$ & $\mathrm{MHC}$ & HOME, Prison & 1.5 \\
\hline Consultation with GP & 1 & MD & $75^{\prime}$ & $\mathrm{MHC}$ & - & \\
\hline $\begin{array}{l}\text { Consultation with neuropsychiatric chilDCCood and adolescence } \\
\text { services }\end{array}$ & 1.3 & MD & $75^{\prime}$ & MHC & - & \\
\hline Inpatient consultation & 1 & MD & $90^{\prime}$ & $\mathrm{H}$ & - & \\
\hline Consultation with PDD & 1.2 & MD & $75^{\prime}$ & COM - LHT & $\mathrm{MHC}$ & 1 \\
\hline Other LHA Department Consultation & 1.2 & MD & $75^{\prime}$ & COM - LHT & - & \\
\hline Staff discussion & 1.8 & MD-N & $60^{\prime}$ & MHC & DC & 1 \\
\hline Written/oral report for other LHA Departments & 1.5 & RT-AS & $60^{\prime}$ & COMMUNITY & - & \\
\hline Written/oral report for other MH-PDD-Departments & 1.9 & SA & $60^{\prime}$ & MHC & DC & 1 \\
\hline Drug supply & 1.1 & $\mathrm{~N}$ & $30^{\prime}$ & MHC & - & \\
\hline Drip & 1.2 & $\mathrm{~N}$ & $40^{\prime}$ & MHC & DC & 1 \\
\hline Vocational training & 1.6 & RT-Ot & $60^{\prime}$ & DC & - & \\
\hline Group psychoeducation (family) & 1.5 & MD-PSY & $55^{\prime}$ & MHC & - & 0.36 \\
\hline Individual psychoeducation (family) & 1.4 & MD-PSY & $60^{\prime}$ & HOME & MHC & 0.67 \\
\hline Group psychoeducation (patient) & 1.3 & N-RT & $55^{\prime}$ & MHC & - & 0.36 \\
\hline Individual psychoeducation (patient) & 1.5 & MD-N & $90^{\prime}$ & MHC & - & \\
\hline Intervention to solve social or administrative problems & 1.2 & $\mathrm{~N}$ & $75^{\prime}$ & MHC & COM - LHT, HOME & 1.5 \\
\hline Group socio-rehabilitation intervention & 2.2 & $\mathrm{~N}-\mathrm{Ot}$ & $30^{\prime}$ & DC & COMMUNITY & 0.36 \\
\hline Individual socio-rehabilitation intervention & 1.9 & N-RT & $60^{\prime}$ & DC & - & \\
\hline Group motor-espressive intervention & 2.2 & $\mathrm{~N}$ & $55^{\prime}$ & $\mathrm{DC}$ & - & 0.36 \\
\hline Individual. motor-espressive intervention & 2.2 & $\mathrm{~N}$ & $75^{\prime}$ & DC & - & \\
\hline Network intervention & 1.7 & $\mathrm{~N}$ & $60^{\prime}$ & DC & MHC & 1 \\
\hline Group socialization intervention & 2.2 & N-RT-Ot & $30^{\prime}$ & DC & COMMUNITY & 0.36 \\
\hline Individual socialization intervention & 2.1 & N-RT & $90^{\prime}$ & DC & - & \\
\hline Social and health support & 2.1 & $\mathrm{~N}$ & $60^{\prime}$ & $\mathrm{DC}$ & COM - LHT, HOME & 1.5 \\
\hline Online clinical referral to GPs & 1.3 & MD-Ot & $15^{\prime}$ & MHC & - & \\
\hline Hospital referral & 1.4 & MD-N & $50^{\prime}$ & MHC & $\mathrm{H}$ & 1.5 \\
\hline Non-hospital compulsory psychiatric treatment & 1.9 & MD-N & $45^{\prime}$ & $\begin{array}{l}\text { COM - LHA, } \\
\text { HOME }\end{array}$ & - & \\
\hline Hospital compulsory psychiatric treatment & 2 & MD-N & $120^{\prime}$ & $\begin{array}{l}\text { COM - LHA, } \\
\text { HOME }\end{array}$ & $\mathrm{H}$ & 1 \\
\hline Couple psychotherapy & 1 & PSY & $50^{\prime}$ & MHC & - & \\
\hline Group psychotherapy & 2.2 & MD-N & $90^{\prime}$ & COMMUNITY & MHC & 0.36 \\
\hline Family Psychotherapy & 1.2 & PSY-SA & $45^{\prime}$ & MHC & - & \\
\hline Individual psychotherapy & 1 & PSY & $60^{\prime}$ & MHC & - & \\
\hline Group holiday & 2.8 & Ot & $330^{\prime}$ & COMMUNITY & - & 0.36 \\
\hline Individual holiday & 2.1 & N-RT & $330^{\prime}$ & $\mathrm{DC}$ & - & \\
\hline Drug administration & 1.8 & $\mathrm{~N}$ & $30^{\prime}$ & $\mathrm{DC}$ & MHC, HOME & 1.5 \\
\hline Standardized assessment & 1.2 & PSY & $40^{\prime}$ & $\mathrm{MHC}$ & - & \\
\hline Grand Total & 1.7 & - & $60^{\prime}$ & - & - & - \\
\hline
\end{tabular}

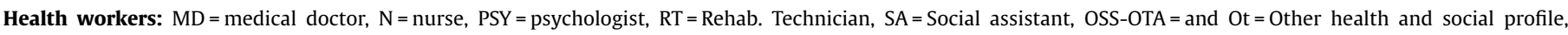
$\mathrm{GP}=$ general practitioner.

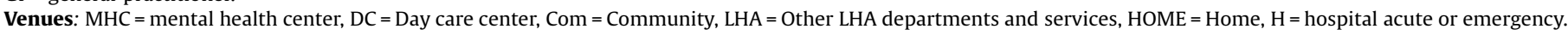

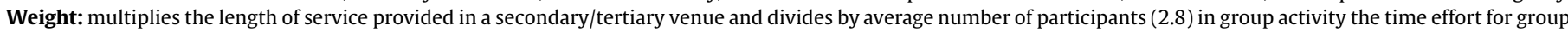
activities $(1: 2.8=0.36)$.

were not included. Pathway costs were calculated as the sum of unit costs of services provided within each specific care pathway.

\subsection{Statistical analysis}

Patients' characteristics were summarized and compared between 2 groups of patients: high-cost (HC), defined as those in the top decile of the cost distribution and non-high-cost (non$\mathrm{HC}$ ), including patients in 1st to 9th deciles of cost distribution.

Comparisons between groups were performed using MannWhitney test or $t$-test for continuous variables when appropriate (age, years in contact with $\mathrm{MH}$ services) and $\chi^{2}$-test for categorical variables.
Multiple linear regression models were used to identify the demographic and clinical predictors of community mental health care costs. The natural log of costs was taken as the dependent variable in the models to normalize the cost distribution, that was skewed to the right. Potential predictors were selected based on their known potential association with the outcome, consistent with Jones et al. [15] and with a previous study of our group underscoring disparities in the use of mental health services between Italians and immigrants [16]. They included gender, age, citizenship (Italian coded as 0 and non-Italian coded as 1 ), education, living arrangement and occupation and number of years since first contact with mental health services and diagnosis. Specifically, eight dummy variables were used to denote the presence or absence of eight primary or 
Table 2

Gross hourly average labor cost by main qualification of health workers in the selected LHA, year 2013.

\begin{tabular}{|c|c|c|c|c|c|c|c|c|}
\hline \multirow[b]{2}{*}{ Qualification } & \multirow[b]{2}{*}{ Contract levels } & \multicolumn{3}{|c|}{ Average gross labor cost } & \multirow{2}{*}{$\begin{array}{l}\text { B } \\
\text { N. of full-time } \\
\text { equivalent staff }\end{array}$} & \multirow{2}{*}{$\begin{array}{l}\mathrm{C} \\
(\mathrm{A} * \mathrm{~B}) \\
\text { Total gross Sum of } \\
\text { wages and on-costs }\end{array}$} & \multirow{2}{*}{$\begin{array}{l}\text { D } \\
(1915,2 * \text { B }) \\
\text { Yearly contract } \\
\text { hrs * FTE }\end{array}$} & \multirow{2}{*}{$\begin{array}{l}\text { E } \\
\text { C/D } \\
\text { Cost per } \\
\text { hour }\end{array}$} \\
\hline & & Min. & Max. & Av. & & & & \\
\hline Nurse & 16 & $€ 38949$ & $€ 46513$ & $€ 41761$ & 223.93 & $€ 9351590$ & 428871 & $€ 21.8$ \\
\hline $\begin{array}{l}\text { Medical doctor (psychiatrist, internist } \\
\text { and toxicologist) }\end{array}$ & 20 & $€ 90292$ & $€ 123519$ & $€ 95773$ & 97.05 & $€ 9294750$ & 192508 & $€ 48.3$ \\
\hline Psychologist & 9 & $€ 72267$ & $€ 86851$ & $€ 76323$ & 58.71 & $€ 4480895$ & 116457 & $€ 38.5$ \\
\hline Social-health educator & 8 & $€ 40076$ & $€ 46513$ & $€ 42734$ & 51.33 & $€ 2193555$ & 98307 & $€ 22.3$ \\
\hline Health assistant (OSS) & 6 & $€ 33616$ & $€ 35935$ & $€ 34961$ & 51.02 & $€ 1783690$ & 97714 & $€ 18.3$ \\
\hline Logopedist & 7 & $€ 38949$ & $€ 44198$ & $€ 41516$ & 34.95 & $€ 1450969$ & 66936 & $€ 21.7$ \\
\hline Physiotherapist & 6 & $€ 38949$ & $€ 43999$ & $€ 41769$ & 18.24 & $€ 761871$ & 34933 & $€ 21.8$ \\
\hline Psychiatric rehabilitation technician & 3 & $€ 38949$ & $€ 44198$ & $€ 41170$ & 18.5 & $€ 761645$ & 35431 & $€ 21.5$ \\
\hline Social worker & 5 & $€ 38949$ & $€ 40076$ & $€ 39043$ & 6.57 & $€ 256513$ & 12583 & $€ 20.4$ \\
\hline Technical assistant (OTA) & 4 & $€ 33616$ & $€ 34356$ & $€ 34208$ & 4.83 & $€ 165225$ & 9250 & $€ 17.9$ \\
\hline Orthoptist & 1 & $€ 44198$ & $€ 44198$ & $€ 44198$ & 2.16 & $€ 95468$ & 4137 & $€ 23.1$ \\
\hline Health care assistant & 2 & $€ 43149$ & $€ 46513$ & $€ 44831$ & 2 & $€ 89662$ & 3830 & $€ 23.4$ \\
\hline Dietician & 1 & $€ 40076$ & $€ 40076$ & $€ 40076$ & 0.5 & $€ 20038$ & 958 & $€ \mathbf{2 0 . 9}$ \\
\hline Radiotechnician & 1 & $€ 44198$ & $€ 44198$ & $€ 44198$ & 1 & $€ 44198$ & 1915 & $€ 23.1$ \\
\hline Tot./min-max-av. & 89 & $€ 33616$ & $€ 123519$ & $€ 47326$ & 570.79 & $€ 30750067$ & 1103831 & $€ 27.9$ \\
\hline
\end{tabular}

*1 Medical doctor and 1 Psychologist FTE $=1983.6$ and any other 1 heath professional FTE $=1915.2 \mathrm{~h}$.

secondary diagnoses: psychosis, bipolar disorder, depression, anxiety, personality disorder, substance use disorder, dementia and other diseases. Three relevant socio-demographic variables (education, living arrangement and occupation) were not included in the models because the percentage of missing data was high (respectively $25.0 \%, 24.9 \%$ and $56.1 \%$ ). All analyses were performed using IBM SPSS, version 23.

Table 3

Patient characteristics $(\mathrm{N}=7601)$.

\begin{tabular}{|c|c|c|c|c|c|c|}
\hline & $\mathrm{N}$ & $\%$ & $\mathrm{HC}(\mathrm{n}=764) \%$ & Non-HC $(n=6837)$ & Test, p & \\
\hline \multicolumn{7}{|l|}{ Sex } \\
\hline Males & 3.155 & 41.5 & 50.8 & 40.5 & 30.11 & $<0.001$ \\
\hline Females & 4.446 & 58.5 & 49.2 & 59.5 & & \\
\hline \multicolumn{7}{|l|}{ Citizenship } \\
\hline Italian & 7.127 & 93.8 & 95.4 & 93.6 & 3.84 & 0.05 \\
\hline Other & 472 & 6.2 & 4.6 & 6.4 & & \\
\hline missing & 2 & & & & & \\
\hline \multicolumn{7}{|l|}{ Education } \\
\hline Primary & 3.702 & 65.0 & 65.1 & 65.0 & 7.64 & $<0.05$ \\
\hline Secondary & 1.667 & 29.3 & 31.2 & 29.0 & & \\
\hline University degree & 329 & 5.8 & 3.7 & 6.1 & & \\
\hline Missing & 1.903 & & & & & \\
\hline \multicolumn{7}{|l|}{ Employment status } \\
\hline Unemployed & 3209 & 96.1 & 99.6 & 95.5 & 18.44 & $<0.001$ \\
\hline Employed & 131 & 3.9 & 0.4 & 4.5 & & \\
\hline missing & 4.261 & & & & & \\
\hline \multicolumn{7}{|l|}{ Living arrangement } \\
\hline own family or cohabiting & 4.847 & 84.9 & 84.6 & 85.0 & 6.05 & 0.195 \\
\hline alone & 744 & 13.0 & 12.5 & 13.1 & & \\
\hline health social housing & 106 & 1.9 & 2.9 & 1.7 & & \\
\hline homeless & 8 & 0.1 & 0 & 0.2 & & \\
\hline prison & 1 & 0 & 0 & 0 & & \\
\hline missing & 1895 & & & & & \\
\hline \multicolumn{7}{|l|}{ Diagnosis } \\
\hline Psychosis & 1693 & 22.3 & 52.4 & 18.9 & 444.0 & $<0.001$ \\
\hline Bipolar disorder & 430 & 5.7 & 8.1 & 5.4 & 9.61 & $<0.01$ \\
\hline Depression & 2337 & 30.7 & 12.2 & 32.8 & 137.61 & $<0.001$ \\
\hline Anxiety & 1378 & 18.1 & 8.2 & 19.2 & 55.90 & $<0.001$ \\
\hline Dementia & 312 & 4.1 & 2.1 & 4.3 & 8.72 & $<0.01$ \\
\hline Alcohol/substance use disorder & 331 & 4.4 & 5.8 & 4.2 & 4.02 & $<0.05$ \\
\hline personality disorder & 1203 & 15.8 & 21.1 & 15.2 & 17.55 & $<0.001$ \\
\hline other $\mathrm{MH}$ & 422 & 5.6 & 8.8 & 5.2 & 16.77 & $<0.001$ \\
\hline adjustment disorder & 655 & 8.6 & 3.3 & 9.2 & 30.81 & $<0.001$ \\
\hline User type & & & & & 195.55 & $<0.001$ \\
\hline incident & 1813 & 23.9 & 3.4 & 26.1 & & \\
\hline prevalent & 5788 & 76.1 & 96.6 & 73.9 & & \\
\hline \multirow[t]{2}{*}{ Age } & Mean & Median & Median & Median & Test & $\mathrm{P}$ \\
\hline & 51.6 & 51 & 46 & 51 & $t$-test & $<0.001$ \\
\hline Years in contact with MHS & 7.8 & 5.4 & 12.5 & 4.6 & M-W & $<0.001$ \\
\hline Days in contact with MHD in 2013 & 14 & 6 & 48 & 5 & M-W & $<0.001$ \\
\hline n. of MH services & 36.1 & 9 & 106 & 7 & $\mathrm{M}-\mathrm{W}$ & $<0.001$ \\
\hline MH total cost per patient & $€ 2243$ & $€ 236$ & $€ 4238$ & $€ 190$ & $\mathrm{M}-\mathrm{W}$ & $<0.001$ \\
\hline
\end{tabular}


Table 4

MH services unit costs in 2013: key descriptive statistics.

\begin{tabular}{|c|c|c|c|c|}
\hline Service & Av. cost per service & Min. & Max. & StdDev \\
\hline Medical assessment & $€ 44.02$ & $€ 22.85$ & $€ 113.51$ & $€ 9.5$ \\
\hline Compulsory health assessment & $€ 52.58$ & $€ 25.66$ & $€ 102.90$ & $€ 19.8$ \\
\hline Informal care $<4 \mathrm{~h}$ & $€ 152.99$ & $€ 56.28$ & $€ 374.82$ & $€ 73.4$ \\
\hline Informal care $>4 \mathrm{~h}$ & $€ 364.09$ & $€ 143.38$ & $€ 735.53$ & $€ 136.1$ \\
\hline Internistic assessment & $€ 22.91$ & $€ 8.30$ & $€ 38.98$ & $€ 8.2$ \\
\hline Group discussion & $€ 81.09$ & $€ 28.14$ & $€ 187.41$ & $€ 36.5$ \\
\hline Family interview & $€ 50.22$ & $€ 24.42$ & $€ 179.11$ & $€ 18.2$ \\
\hline Phone call $>15 \min$ & $€ 31.46$ & $€ 15.15$ & $€ 32.48$ & $€ 4.2$ \\
\hline Individual interview/consultation & $€ 46.50$ & $€ 21.98$ & $€ 174.17$ & $€ 17.8$ \\
\hline Consultation with GP & $€ 45.23$ & $€ 24.89$ & $€ 90.88$ & $€ 3.7$ \\
\hline Consultation with neuropsychiatric childhood and adolescence services & $€ 86.00$ & $€ 75.73$ & $€ 116.81$ & $€ 19.0$ \\
\hline Inpatient consultation & $€ 63.21$ & $€ 36.93$ & $€ 126.48$ & $€ 2.7$ \\
\hline Consultation with PDD & $€ 51.88$ & $€ 25.71$ & $€ 115.76$ & $€ 19.5$ \\
\hline Other LHA Department Consultation & $€ 51.61$ & $€ 25.71$ & $€ 174.17$ & $€ 21.0$ \\
\hline Staff discussion & $€ 51.54$ & $€ 19.54$ & $€ 161.56$ & $€ 33.9$ \\
\hline Written/oral report for other LHA Departments & $€ 50.80$ & $€ 31.71$ & $€ 69.88$ & $€ 27.0$ \\
\hline Written/oral report for other MH-PDD-Departments & $€ 72.57$ & $€ 50.47$ & $€ 178.51$ & $€ 40.3$ \\
\hline Drug supply & $€ 9.36$ & $€ 7.33$ & $€ 46.52$ & $€ 3.6$ \\
\hline Drip & $€ 10.92$ & $€ 8.30$ & $€ 31.66$ & $€ 5.8$ \\
\hline Vocational training & $€ 53.31$ & $€ 32.86$ & $€ 67.14$ & $€ 16.3$ \\
\hline Group psychoeducation (family) & $€ 76.23$ & $€ 32.99$ & $€ 167.47$ & $€ 33.7$ \\
\hline Individual psychoeducation (family) & $€ 68.98$ & $€ 33.18$ & $€ 195.80$ & $€ 27.3$ \\
\hline Group psychoeducation (patient) & $€ 43.04$ & $€ 32.59$ & $€ 98.56$ & $€ 20.6$ \\
\hline Individual psychoeducation (patient) & $€ 72.12$ & $€ 29.30$ & $€ 187.53$ & $€ 29.0$ \\
\hline Intervention to solve social or administrative problems & $€ 57.98$ & $€ 39.64$ & $€ 234.42$ & $€ 32.4$ \\
\hline Group socio-rehabilitation intervention & $€ 73.09$ & $€ 28.14$ & $€ 167.17$ & $€ 28.4$ \\
\hline Individual socio-rehabilitation intervention & $€ 63.98$ & $€ 29.30$ & $€ 187.53$ & $€ 24.1$ \\
\hline Group motor-espressive intervention & $€ 61.91$ & $€ 22.51$ & $€ 133.73$ & $€ 23.3$ \\
\hline Individual. motor-espressive intervention & $€ 79.51$ & $€ 29.30$ & $€ 160.90$ & $€ 28.3$ \\
\hline Network intervention & $€ 65.67$ & $€ 29.30$ & $€ 242.34$ & $€ 31.3$ \\
\hline Group socialization intervention & $€ 57.39$ & $€ 22.51$ & $€ 149.93$ & $€ 22.6$ \\
\hline Individual socialization intervention & $€ 51.65$ & $€ 21.98$ & $€ 153.62$ & $€ 14.3$ \\
\hline Social and health support & $€ 74.47$ & $€ 29.30$ & $€ 186.06$ & $€ 37.7$ \\
\hline Online clinical referral to GPs & $€ 5.31$ & $€ 2.86$ & $€ 9.25$ & $€ 2.0$ \\
\hline Hospital referral & $€ 62.39$ & $€ 27.38$ & $€ 156.01$ & $€ 21.7$ \\
\hline Non-hospital compulsory psychiatric treatment & $€ 75.30$ & $€ 45.44$ & $€ 115.76$ & $€ 18.3$ \\
\hline Hospital compulsory psychiatric treatment & $€ 224.45$ & $€ 66.36$ & $€ 416.88$ & $€ 94.1$ \\
\hline Couple psychotherapy & $€ 39.72$ & $€ 27.65$ & $€ 69.71$ & $€ 8.5$ \\
\hline Group psychotherapy & $€ 144.72$ & $€ 80.69$ & $€ 240.55$ & $€ 47.5$ \\
\hline Family Psychotherapy & $€ 46.77$ & $€ 31.71$ & $€ 111.06$ & $€ 21.9$ \\
\hline Individual psychotherapy & $€ 37.87$ & $€ 23.78$ & $€ 128.73$ & $€ 4.8$ \\
\hline Group holiday & $€ 404.25$ & $€ 143.38$ & $€ 708.19$ & $€ 160.5$ \\
\hline Individual holiday & $€ 377.64$ & $€ 180.74$ & $€ 532.17$ & $€ 94.9$ \\
\hline Drug administration & $€ 11.36$ & $€ 4.88$ & $€ 34.74$ & $€ 6.4$ \\
\hline Standardized assessment & $€ 71.02$ & $€ 36.63$ & $€ 188.88$ & $€ 26.9$ \\
\hline Grand Total & $€ 61.92$ & $€ 2.86$ & $€ 735.53$ & $€ 75.2$ \\
\hline
\end{tabular}

\section{Results}

\subsection{Study population}

Of the 8699 patients retrieved from the SISM database, 53 were excluded because their diagnosis had not been established yet, and 1045 were further excluded because their diagnosis was recorded using V-codes, that specify an encounter for administrative purposes or a general psychiatric examination. Therefore, the final analyzed population consisted of 7601 patients (Table 3), predominantly female, with a mean age of 51 years and a longstanding relationship with mental health services. The most frequent diagnoses were depression (30.7\%), psychosis $(22.3 \%)$ and personality disorders (15.8\%).

Table 5

Frequency of MH 'pathways' and costs per beneficiary in 2013.

\begin{tabular}{|c|c|c|c|c|c|c|}
\hline & \multirow[t]{2}{*}{$\mathrm{N}$ of patients } & \multirow[t]{2}{*}{$\%$ of total patients } & \multirow[t]{2}{*}{ Tot.MH-PDD costs } & \multirow[t]{2}{*}{$\%$ of total costs } & \multicolumn{2}{|c|}{ Costs per patient } \\
\hline & & & & & Mean & Median \\
\hline Day center & 290 & 3.8 & $€ 8940029$ & 52.4 & $€ 30827.69$ & $€ 11997.16$ \\
\hline Psychiatric-clinical & 5044 & 66.4 & $€ 3484245$ & 20.4 & $€ 690.77$ & $€ 377.11$ \\
\hline Community day-hospital & 104 & 1.4 & $€ 2648490$ & 15.5 & $€ 25466.26$ & $€ 20948.49$ \\
\hline Socio-rehabilitation & 307 & 4.0 & $€ 1406828$ & 8.3 & $€ 4582.50$ & $€ 1678.40$ \\
\hline Initial (re)assessment & 3560 & 46.8 & $€ 413470$ & 2.4 & $€ 116.14$ & $€ 98.23$ \\
\hline Psychotherapy & 295 & 3.9 & $€ 99136$ & 0.6 & $€ 336.05$ & $€ 227.12$ \\
\hline Vocational training & 200 & 2.6 & $€ 58990$ & 0.3 & $€ 294.95$ & $€ 187.12$ \\
\hline Total & & & $€ 17051188$ & 100.0 & & \\
\hline
\end{tabular}




\subsection{Costs}

The overall costs of the $274639 \mathrm{MH}-\mathrm{PDD}$ services provided in 2013 amounted to more than 17 million $€$. Estimated unit costs varied from a minimum of $€ 2.86$ to a maximum of $€ 735.53$, which respectively corresponded to a health-allied professional performing a 5 min task, and to an informal access to a day care facility which lasted more than $4 \mathrm{~h}$ and involved up to 4 health professionals (Table 4). Some unit costs had higher variance when infrequent and when performed by very different skill-mixes.

Pathway costs are displayed in Table 5. The cost methodologies defined for MH services imply that treatment costs increase with the duration and the number of operators involved. THCs sociorehabilitation, day center and community day hospital care were by far the most expensive interventions, although they were provided to a minority of patients.

For instance, day center activities accounted for more than $52 \%$ of total costs but involved less than $4 \%$ of patients. Psychotherapy costed annually a median of $€ 227$ and was among the cheapest interventions together with vocational training ( $€ 187)$ and initial assessment (€98).

Fig. 1 shows that the $\mathrm{HC}(\mathrm{N}=764)$ accounted for $87 \%$ of costs and $73 \%$ of services, while the remaining $90 \%$ of patients accounted for only $13 \%$ of costs. The reason for such huge disparity is that the great bulk of costs incurred for HC included day-center, community day hospital and socio-rehabilitation services, that were extremely uncommon in non-HC (Fig. 2).

Comparison of $\mathrm{HC}$ patients with non-HC revealed that $\mathrm{HC}$ included a significantly higher proportion of males with psychosis,

\section{Costs}

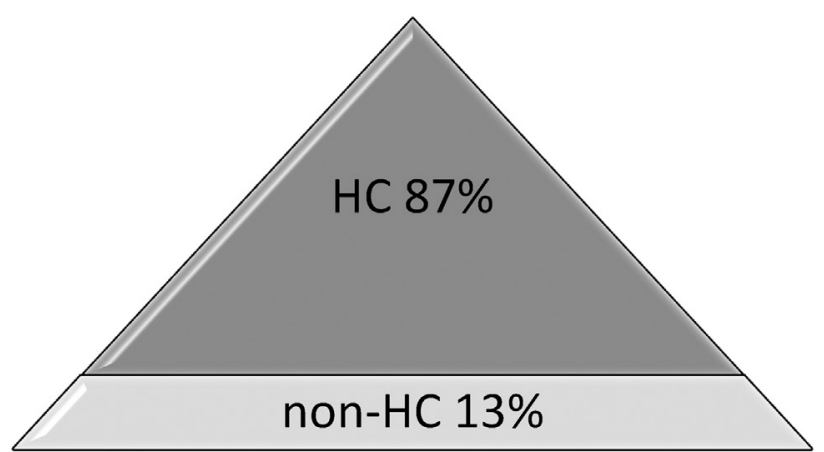

Services

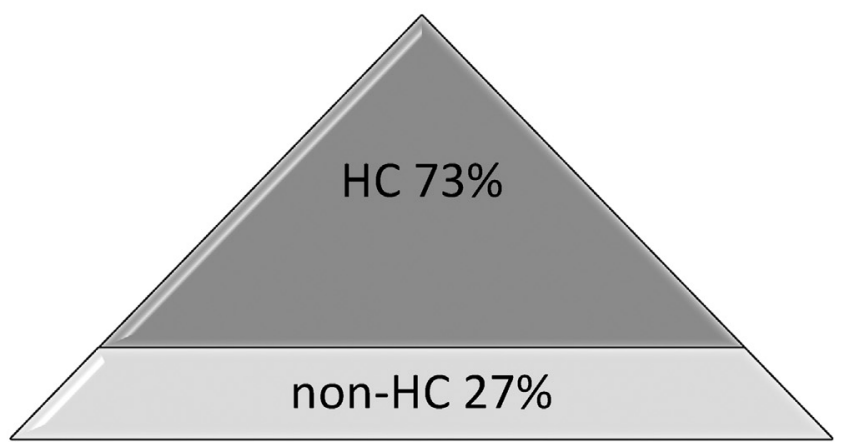

Fig. 1. Diagram showing the distribution of annual costs and services among patients treated at the MH-PDD in 2013.

High cost (HC): annual costs $>€ 1520$; non-high costs (non-HU): annual costs $\leq €$ 1520 . HC accounted for $87 \%$ of costs and $73 \%$ of services.

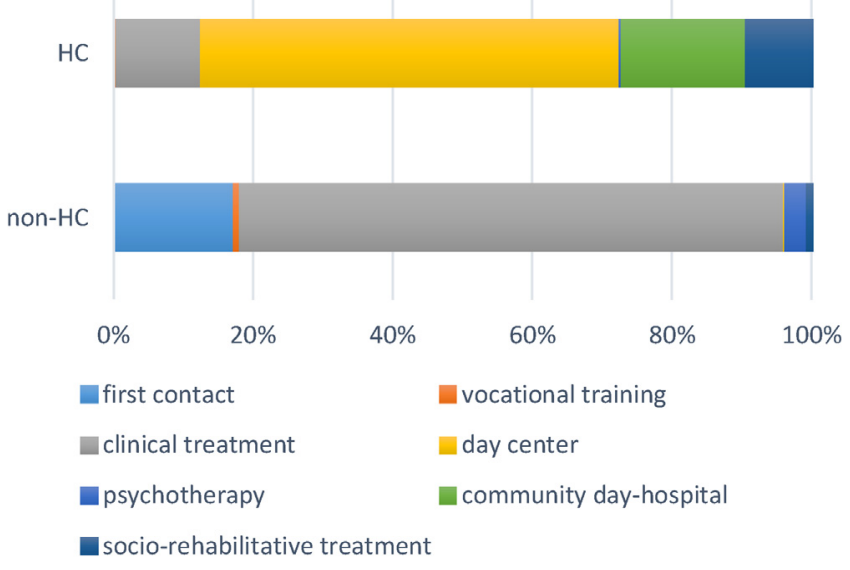

Fig. 2. Stacked bar chart showing the percentage distribution of cost by pathway in the top $10 \%(\mathrm{HC})$ and in the rest of the sample (non-HC).

High cost $(\mathrm{HC}):>€ 1520$; non-HC: $\leq € 1520$.

with a lower median age (46 years), a median of 12.5 years in contact with MHS and a median of 106 services in the index year (Table 4). Vice versa, non-HC $(\mathrm{N}=6837)$ comprised predominantly women with a median age of 51 years, with common mental disorders (depression, anxiety or adjustment disorder), who had been in contact with MHS for 4.6 years and received a median of 7 services; $26.1 \%$ of them were incident cases.

The median cost of $\mathrm{HC}$ patients was about 22-fold higher compared to that of non-HC patients ( $€ 4238$ vs. $€ 190$ ).

Median annual costs were highest for psychosis (€562), although with a large variability (range $€ 8.3-391652$ ), and were $€$ 439 for bipolar disorder (range $€ 8.3$-137239), $€ 214$ for personality disorder (range $€ 8.3-209607$ ), $€ 182$ for depression (range $€ 5-90162$ ).

\subsection{Predictors of costs}

We regressed costs on age and gender and then entered, with a stepwise procedure citizenship, the 8 diagnoses and the duration of contact with MHS (in years). This model accounted for $20.7 \%$ of cost variance. The statistically significant predictors of costs, arranged in decreasing order of weight (standardized beta coefficient), were psychosis, a longer relationship with mental health services, younger age, personality disorder, bipolar disorder, depression, dementia and Italian citizenship (Table 6).

\section{Discussion}

In this study we developed a cost methodology that combines direct and indirect costs of community mental healthcare, and

Table 6

Linear regression estimates of $(\log )$ costs by MH users characteristics $(N=7061)$. The adjusted $R^{2}$ for the model is 0.207 .

\begin{tabular}{lrrrrrr}
\hline & B & Beta & \multicolumn{1}{c}{$\mathrm{t}$} & $\mathrm{p}$ & $95 \% \mathrm{CI}$ & \\
\hline (constant) & 5.816 & & 60.340 & $<0.001$ & 5.627 & 6.005 \\
Gender & 0.028 & 0.009 & 0.894 & 0.371 & -0.033 & 0.088 \\
Age & -0.016 & -0.179 & -15.557 & $<0.001$ & -0.018 & -0.014 \\
Years since 1st contact & 0.042 & 0.231 & 19.490 & $<0.001$ & 0.038 & 0.046 \\
Psychosis & 1.086 & 0.306 & 23.569 & $<0.001$ & 0.995 & 1.176 \\
Personality disorder & 0.598 & 0.148 & 13.052 & $<0.001$ & 0.508 & 0.688 \\
Bipolar disorder & 0.797 & 0.125 & 11.373 & $<0.001$ & 0.659 & 0.934 \\
Depression & 0.232 & 0.072 & 5.863 & $<0.001$ & 0.154 & 0.309 \\
Dementia & 0.325 & 0.044 & 3.973 & $<0.001$ & 0.165 & 0.486 \\
Citizenship & -0.150 & -0.025 & -2.331 & 0.020 & -0.276 & -0.024 \\
\hline
\end{tabular}


applied it to a cohort of patients treated in 2013 by the MH-PDD of an Italian local health authority to estimate the cost of services and care pathways. Day center was by far the more expensive pathway, accounting for $52.4 \%$ of the total annual costs, although it was used by a minority of patients.

Notably, our findings indicated that mental healthcare costs are concentrated among a small fraction of patients (10\% of patients generating $87 \%$ of costs), suffering from severe mental illness, relatively younger than the rest of the patients and with an established relationship with mental health services. This suggests that most expensive interventions are targeted to complex and younger patients, in the attempt to counteract the burden of illness for patients and their families. In particular, high-cost patients were those who benefitted from care pathways including rehabilitation services delivered in day centers or community day hospitals.

Our results are consistent with evidence from a study conducted in the catchment area of Madrid [17], in which $19.4 \%$ of patients generated $80 \%$ of costs and with the results of a US study of patients with depression [13], in which mean annual costs of high utilizers (top $10 \%$ of costs) were eightfold higher than those of non-high utilizers.

In addition, our finding that a minority of patients account for a disproportionately high share of health care cost is in line with a Canadian study showing that, among high-cost patients, those with a substantial use of mental health services incur in 30\% higher costs [12].

Results from multiple linear regression indicate that severe mental illness (encompassing the diagnoses of psychosis, bipolar disorder, and personality disorder), younger age, a longer relationship with mental health services, and dementia predicted higher annual costs. These findings are consistent with Jones et al. review [15] and underscore the importance of previous psychiatric service utilization as a proxy of chronicity, that proved to be the most significant predictor of costs in Donisi et al. [18].

Our finding concerning the economic burden of patients with psychosis, compared with patients with other conditions, has been well documented in the literature and raises concerns worldwide. In a US study it has been estimated at $\$ 155.7$ billion for the year 2013, including excess direct health care costs of $\$ 37.7$ billion (24\%), direct non-health care costs of $\$ 9.3$ billion (6\%), and indirect costs of $\$ 117.3$ billion (76\%) compared to individuals without schizophrenia. The largest components were excess costs associated with unemployment (38\%), productivity loss due to caregiving (34\%), and direct health care costs (24\%) [19]. De Oliveira et al. [14] underscored that expenditures for patients with chronic psychosis represent a sizeable portion of the Ontario health care budget (3\%). A recent review of 19 studies on the societal cost of schizophrenia [20] reported that the annual healthcare costs vary from \$US 1445 in Thailand to \$US 60630 in Norway.

This study has a number of strengths. First, it is based on the large and heterogeneous real-world population attending the community mental health services of Local Health Authority and has no selection bias. Second, cost estimates are accurate because they rely on information recorded in the SISM database about the professional mix involved in the different activities and (when appropriate) the time spent on traveling to the service location. Another strength of our study lies in the novel bottom-up methodology focused on individual costs and based on the staff and MH-PDD resources actually involved in patients' care. In this way, the annual cost per patient, per care pathway and per diagnostic cluster were obtained.

However, our findings should be interpreted in light of important limitations. First, the retrospective study design prevented from having complete data on diagnoses and on some demographic characteristics such as education, working status and living arrangement that may be important determinants of service use and costs. Second, because hospital, residential and drug costs were not included in the estimation, we underestimated overall mental health costs and the costs of patients mainly treated in subcontracted facilities.

Despite these limitations, the methodology employed exploits routinely collected economic and activity data and offers a replicable cost breakdown procedure to be used in other regional local health authorities for a comprehensive estimate of resource utilization across community health services, provided that information systems collecting data at the same detail are available. To facilitate benchmarking, effective 2012, the Italian Ministry of Health has adopted a unique record layout for the mental health information system. All the regions have the obligation to send their data twice a year using a specific format, to allow the generation of reports and quality of care indicators. Moreover, because the magnitude of mental health cost estimates differs considerably across countries, due to different economic conditions, healthcare systems and widespread methodological heterogeneity among COI studies [21], our results can be useful for cross-national comparisons.

In conclusion, on a policy level, the large heterogeneity and the strong asymmetry in the distribution of individual costs of mental health care argues against the use of 'average' costs to summarize mental health care expenditure, in favor of an analytic breakdown based on individual care pathways. The adoption of a standardized microcosting methodology could also introduce MH-PDD healthcare professionals to clinical governance issues and support them in the definition of personalized health care plans that encompass all services and activities that jointly determine the success in meeting patient's needs. This paves the way for measuring 'value' in term of patient outcomes for each euro spent [22,23]. From the clinical point of view, our results concerning the predictors of higher costs underscore the need of to undertake a systematic collection of symptomatic and functional outcomes of the multifaceted interventions provided by community mental health services to ensure that interventions meet patients' needs and are cost-effective and sustainable.

\section{Acknowledgment}

This research did not receive any specific grant from funding agencies in the public, commercial, or not-for-profit sectors.

\section{Appendix A. Supplementary data}

Supplementary data associated with this article can be found, in the online version, at https://doi.org/10.1016/j.eurpsy.2018.02.001.

\section{References}

[1] World Health Organization, Comprehensive Mental Health Action Plan 20132020, Available at http://apps.who.int/gb/ebwha/pdf_files/WHA66/A66_R8en pdf?ua=1, (Accessed 11 September 2017).

[2] OECD, Making Mental Health Count: The Social and Economic Costs of Neglecting Mental Health Care, Health Policy Studies, Available at http://www. oecd.org/els/health-systems/making-mental-health-count-9789264208445en.htm, (Accessed 11 September 2017)

[3] Decree of the President of the Council of Ministers 12 gennaio 2017: definizione e aggiornamento dei livelli essenziali di assistenza, Available at http://www.trovanorme.salute.gov.it/norme/dettaglioAtto? id=58669\&completo=true, (Accessed September 11 2017).

[4] Regional Decree 2155/2011, Azioni innovative a sostegno delle previsioni del piano attuativo salutementale 2009-2011, (Available upon request at http://servizissiir regione.emilia-romagna.it/deliberegiunta/servlet/AdapterHTTP?action_name= ACTIONRICERCADELIBERE\&operation=leggi\&cod_protocollo=GPG/2011/1808).

[5] Starace F. Manuale pratico per l'integrazione sociosanitaria: il modello del Budget di salute. Collana Carroccifaber Le professioni sanitarie.

[6] Health Ministry, Rapporto salute mentale: analisi dei dati del Sistema informativo per la salute mentale (SISM) anno 2015, Available at http://www. 
salute.gov.it/portale/documentazione/p6 2_2 1.jsp? lingua=italiano\&id=2550, (Accessed September 11, 2017).

[7] Amaddeo F, Grigoletti L, Montagni I. Mental health care financing in Italy: current situation and perspectives. J Nerv Ment Dis 2014:202:464-8.

[8] Mapelli V, De Stefano A, Compagnoni V, Gambino A, Ceccarelli A. I sistemi di governance dei servizi sanitari regionali, Quaderno Formez n. 57. 2007.

[9] Rampazzo L. I costi standard in salute mentale. Salute e Territorio 2014 Fascicolo 199/200.

[10] S.G. Armesto, H. Medeiros, L. Wei, Information Availability for Measuring and Comparing Quality of Mental Health Care Across OECD Countries. OECD Health Technical Papers 2008, 20, (Available at http://www.oecd.org/els/healthsystems/41243838.pdf, accessed September 2, 2008).

[11] Fattore G, Percudani M, Pugnoli C, Contini A, Beecham J. Mental health care in Italy: organisational structure, routine clinical activity and costs of a community psychiatric service in Lombardy region. Int J Soc Psychiatry 2000;46:250-65.

[12] de Oliveira C, Cheng J, Vigod S, Rehm J, Kurdyak P. Patients with high mental health costs incur over 30\% more costs than other high-cost patients. Health Aff (Millwood) 2016;35(January (1)):36-43.

[13] Robinson RL, Grabner M, Palli SR, Faries D, Stephenson JJ. Covariates of depression and high utilizers of healthcare: impact on resource use and costs. J Psychosom Res 2016;85(June):35-43.

[14] de Oliveira C, Cheng J, Rehm J, Kurdyak P. The economic burden of chronic psychotic disorders in Ontario. J Ment Health Policy Econ 2016;19(December (4)):181-92.

[15] Jones J, Amaddeo F, Barbui C, Tansella M. Predicting costs of mental health care: a critical literature review. Psychol Med 2007;37(April (4)):467-77.

[16] Rucci P, Piazza A, Perrone E, Tarricone I, Maisto R, Donegani I, et al. Disparities in mental health care provision to immigrants with severe mental illness in Italy. Epidemiol Psychiatr Sci 2015;24(August (4)):342-52.

[17] S.G. Armesto, H. Medeiros, L. Wei, Information Availability for Measuring and Comparing Quality of Mental Health Care Across OECD Countries. OECD Health Technical Papers 2008, 20, Available at http://www.oecd.org/els/healthsystems/41243838.pdf, (Accessed 2 September 2008).

[18] Donisi V, Jones J, Pertile R, Salazzari D, Grigoletti L, Tansella M, et al. The difficult task of predicting the costs of community-based mental health care: a comprehensive case register study. Epidemiol Psychiatr Sci 2011;20 (September (3)):245-56.

[19] Baca-Garcia E, Perez-Rodriguez MM, Basurte-Villamor I, Quintero-Gutierrez FJ, Sevilla-Vicente J, Martinez-Vigo M, et al. Patterns of mental health service utilization in a general hospital and outpatient mental health facilities: analysis of 365,262 psychiatric consultations. Eur Arch Psychiatry Clin Neurosci 2008;258:117-23.

[20] Cloutier M, Aigbogun MS, Guerin A, Nitulescu R, Ramanakumar AV, Kamat SA, et al. The economic burden of schizophrenia in the United States in 2013. J Clin Psychiat 2016;77:764-71.

[21] Jin H, Mosweu I. The societal cost of schizophrenia: a systematic review. Pharmacoeconomics 2017;35:25-42.

[22] Porter ME. What is value in health care. New Engl J Med 2010;363:2477-81.

[23] Nuti S, Vola F, Bonini A, Vainieri M. Making governance work in the health care sector: evidence from a 'natural experiment' in Italy. Health Econ Policy Law 2016;11:17-38.

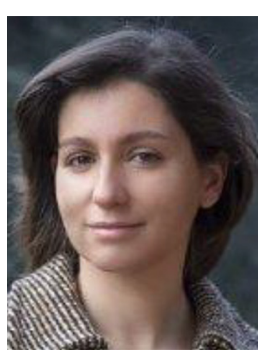

Francesca Senese has a Master degree in Health Economics and Management (University of Bologna) and a University Degree in Cultural Studies (University of Modena and Reggio Emilia).

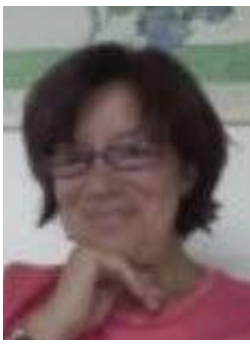

Paola Rucci is a statistician, has a PhD in General Medica and Services Sciences and is Researcher in Medical Statistics at the School of Medicine of the University of Bologna.

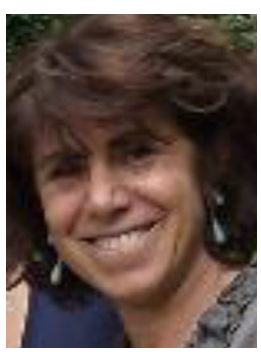

Maria Pia Fantini is Full Professor of Hygiene and Public Health at the University of Bologna.

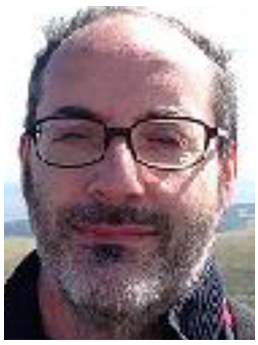

Dino Gibertoni is a statistician, has a $\mathrm{PhD}$ in Genera Medical and Services Sciences and is post-doctoral fellow in Medical Statistics.

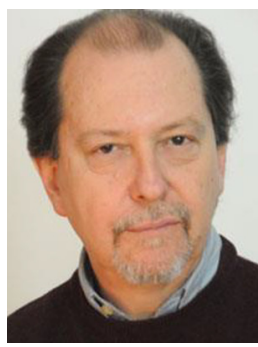

Enrico Semrov is a psychiatrist and Head of the Mental Health Center of Reggio Emilia Nord-East.

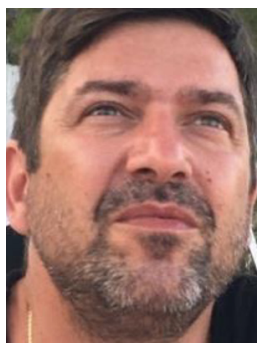

Matteo Nassisi is Head of the mental health information system of the Local Health Authority of Reggio Emilia

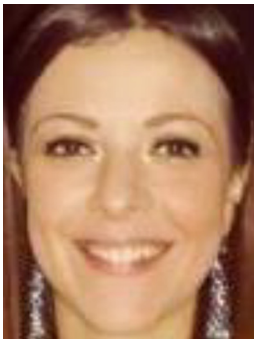

Rossella Messina is a psychologist and a PhD candidate in General Medical and Services Sciences.

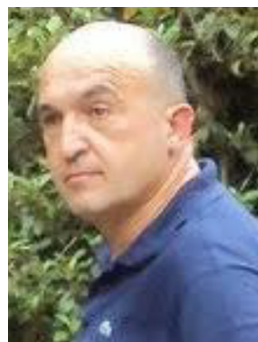

Claudio Travaglini is Full Professor of Business Administration at the University of Bologna. 